\title{
A Study of Fault-tolerant Design of Chinese Characters Under the Gestalt Organization Law
}

\author{
Zhixiong Huang ${ }^{1, *}$ Kexuan Wang ${ }^{1}$
}

\author{
${ }^{1}$ Fuzhou University, Fuzhou, Fujian, China \\ *Corresponding author. Email: 38501093@qq.com
}

\begin{abstract}
Gestalt organization law is closely related to design science. It is a specific organizational law when human vision perceives the outside world, thus it is widely used in visual arts. This article discusses the "fault-tolerant" design of Chinese characters based on the Gestalt organization law, aiming to dig out the hidden features and artistic expressions of Chinese characters under the Gestalt organization law and draw the laws of fault-tolerant design of Chinese characters, so that the design of Chinese characters has creative expressions of differences and unique connotations.
\end{abstract}

Keywords: Obtaining "meaning" and forgetting "form", Gestalt organization law, Chinese character fault-tolerant design.

\section{INTRODUCTION}

In the current era of design change, a unified standard font can no longer show the inner implication and individuality of Chinese characters. Gestalt psychologist Wertheimer acquiesced that: "In the process of visual perception, individuals always have a tendency to pursue the integrity of things unconsciously, and this tendency refers to what they call Gestalt" $[1]^{132}$. He also called the Gestalt organization law the "graphic optimization trend law", namely, any shape is an organization or structure in experience, and under its influence, the human visual perception organization will try to achieve the best perfect effect. Under the principle of not undermining the identifiability of Chinese characters, it is needed to let the Gestalt organization law speak in the design of Chinese characters, coordinate the relationship between the form and semantics of Chinese characters, deepen and expand the expression and application of the Gestalt law in the design of Chinese characters, and enrich the meaning of Chinese characters.

\section{THE RELATIONSHIP BETWEEN THE GESTALT ORGANIZATION LAW AND THE FAULT TOLERANCE OF CHINESE CHARACTERS}

As a symbol that carries information, Chinese characters require rigorous structure and standardized strokes. However, in the design, in order to pursue the effect of differentiation and uniqueness, appropriate "visual errors" in Chinese characters are allowed, and partial alienation or incompleteness of Chinese characters occurs. This kind of error is a deliberate mistake of subjective consciousness, making the text break through the original constraints without affecting the recognition and information transmission function, and present new connotations and meanings. Therefore, the fault tolerance of Chinese characters is an important feature when recognizing Chinese characters. However, individuals have different experience in Chinese character recognition and cannot be measured by standards. Gestalt organization law, as a kind of visual perception law, allows viewers to automatically recognize or perceive images that are alienated or not fully presented through Gestalt laws such as closure and connection, and then obtain information. Therefore, the Gestalt organization law is the supporting point 
of the fault-tolerant design of Chinese characters. The combination of the two makes the fault-tolerant design of Chinese characters not only consider its own formation characteristics, but also take care of the visual perception rules and psychological characteristics of the viewer, making Chinese character design a modern visual art that combines art and science.

\section{THE GESTALT PHENOMENON IN THE CREATIVE DESIGN OF CHINESE CHARACTERS}

The Gestalt phenomenon in the creative design of Chinese characters is the scientific dismantling and reorganization and rational change of the "form" of Chinese characters after in-depth research and analysis of the form or semantics of the characters, and then to combine it with the "meaning". Under this circumstance, the viewer will have a new visual experience that is both familiar and unfamiliar to the new "form", breaking through the viewer's habitual perceptual experience, and understanding the designer's intentions [1] ${ }^{132}$. The Chinese characters presented to people at this time are no longer constrained by the familiar writing of Chinese characters, but more on the expression of their artistic connotations. As the Japanese designer Kenya Hara wrote, "Defining a thing or writing it down does not necessarily mean understanding it. If we can first defamiliarize what is already known, and then try to challenge its authenticity, then it will be possible for us to understand it in depth" [2] $]^{1}$.

\subsection{Obtaining "Meaning" and Forgetting "Form" in Calligraphy}

In Chinese calligraphy, there is a saying that "Whatever meaning one wishes to express, his pen follows; obtaining meaning and forgetting form" (" 意在笔先，得意忘形 "), " 意 " refers to artistic conception, that is, style and romantic charm, and " 形" refers to structure and technique of writing. Calligraphers often pursue the inner artistic conception too much in order to express the "tones outside the strings" that are not directly related to Chinese characters or to express the beauty of calligraphy and the beauty of art of composition, and then paint a distinctive stylized image. This phenomenon is reasonable in creation, which is a way of expression for calligraphers to show their elegant taste. For example, in "Figure 1," Ji Xiaolan wrote a couplet for the Confucius Family Mansion and deliberately wrote two words wrong. The word "富" in the first line of the couplet missed a "dot stroke" in "ウ", and the word "章" in the second line of the couplet had an extra stroke running through the word "立", meaning "endless riches and honour" and "articles connecting the sky", reflecting the identity of the Confucian family and low-key publicity. In "Figure 2", the Emperor Kangxi of Qing Dynasty respected the saying that three dot strokes represent water and four dot strokes represent fire in Chinese characters. In the inscription of "Fish Viewing at the Flower Pond", he deliberately wrote the traditional Chinese character "魚" with three dot strokes, expressing Kangxi's compassion and love for all things on earth. In "Figure 3", Xianfeng deliberately removed one rightward stroke from the word "院", thinking that people should be grateful. However, it was difficult to requite all the favors during one's lifetime, so the strokes of the word "完" couldn't be written in full. Wrongly written characters like these are wrong appropriately, which become good stories with the passage of time.

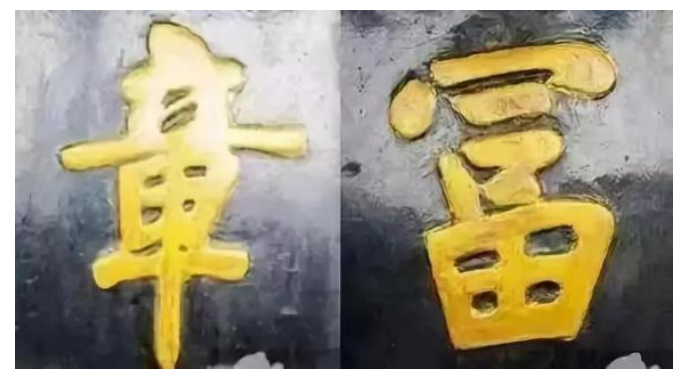

Figure 1 Ji Xiaolan: The couplet at the gate of the Confucius Family Mansion.

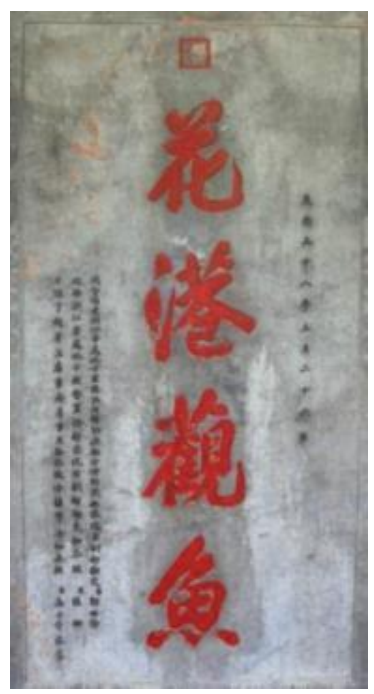


Figure 2 Emperor Kangxi of the Qing Dynasty: The inscription for a scenery plaque.

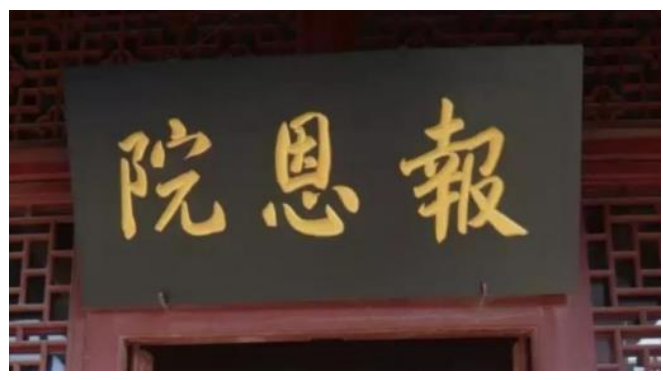

Figure 3 The inscription by Emperor Xianfeng.

\subsection{The Complementary Relationship Between "Form" and "Meaning"}

The concrete external appearance of Chinese characters and the abstract inner meaning are complementary and integrated. Form is the vivid expression of meaning, and meaning is the internal expression of form, that is, "form" is produced by "meaning", and "meaning" is expressed by "form". Although the external appearance of Chinese characters is alienated, it can be supplemented by the inner meaning of Chinese characters to make them blend together organically, show a more unique visual artistic conception, deeper emotions and richer semantics, and then impress the viewers to achieve the visual effect of seeing the form and knowing the meaning. During the bourgeoisdemocratic revolution in the 20th century, a large number of newspapers with the word "民" appeared such as the "《民生日报》" and "《中华民报》". In the headlines of these newspapers, the left-falling stroke of the word "民" was written beyond the word itself, expressing Chinese people's unwillingness to endure the humiliation and torture, and their willingness to stand up and get ahead ("Figure 4"). In Digest News in 2010, a cartoon was eyecatching, in which the word "夫" was written upside down as a symbol of money, being a metaphor for the relationship between husband and money in marriage ("Figure 5") [3 $]^{98}$. In Liu Bingke's font design - instant amnesia, he combined with the lost and incomplete meaning expressed behind it, and subtracted at the partial level of the font to make it show a missing sense of tableau ("Figure $6 ")$. Although these designs reduce the recognition of Chinese characters, they invisibly increase the visual charm and emotional rendering power of Chinese characters, allowing viewers to trigger association mechanisms through missing or alienated forms to get the connotation of meaning, understand the thoughts and emotions behind them, convey the meaning of Chinese characters more clearly and deeply, and perfectly reflect the integration and unity of the form and meaning of Chinese characters.

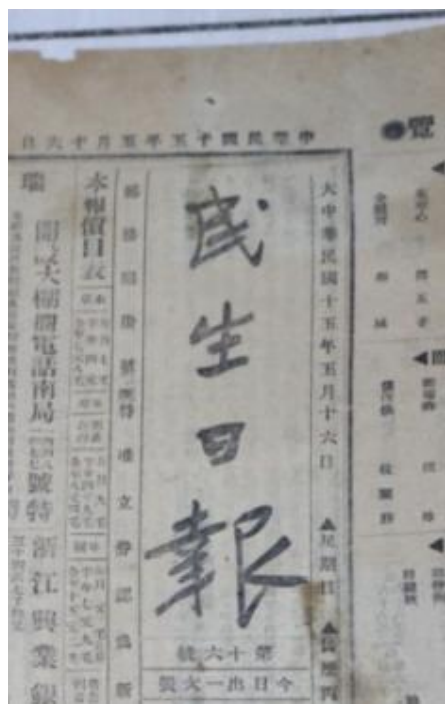

Figure 4 《民生日报》.

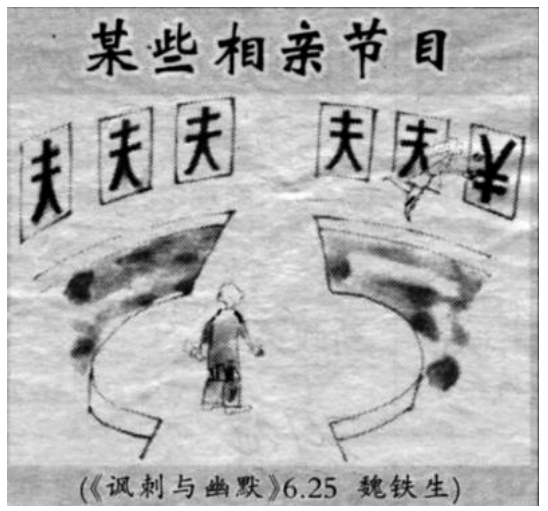

Figure 5 Wei Tiesheng: Irony and Humor. 


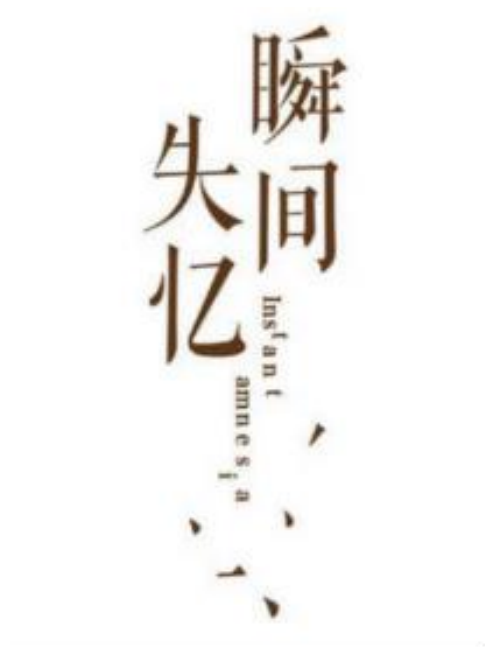

Figure 6 Liu Bingke: Font design.

\section{THE PATTERN OF MANIFESTATION OF THE GESTALT ORGANIZATION LAW IN THE FAULT-TOLERANT DESIGN OF CHINESE CHARACTERS}

The Gestalt organization law deals with the relationship between the whole and the parts, so that the whole presents a more perfect effect $[4]^{49}$. It includes the law of continuity, the law of closure, the law of similarity, the law of proximity, and the law of figure and ground. In font design, the various laws of Gestalt sometimes accompany each other. It combines various laws to process and change the strokes, structures, parts, etc. of Chinese characters, attracting visual attention and making the font design present a better visual effect.

\subsection{The Closure Makes up for the Lack- The Omission and Disconnection of Strokes}

In the long development process of Chinese characters, the most important form of expression is the simplification of stroke and glyph. It intentionally disconnects the closed strokes, strokes connections or intersections, resulting in incomplete Chinese characters. But when people read, they will spontaneously treat multiple disconnected or deleted strokes as a closed pattern according to experience $[4]^{93}$. The missing or disconnected part will not affect the overall recognition of Chinese characters. Instead, it presents the characteristics of being concise, clean and tidy, which better attracts the attention of viewers and triggers visual attention and tension. When reduced, it is necessary to grasp the main form of Chinese characters, grasp the main structure, appropriately retain the relatively special or prominent radicals, appropriately subtract the strokes or parts that are less recognizable or irregular, and organize the whole to achieve a unified beauty of order.

In the design of Chinese characters in "Figure 7", many cross strokes are simplified and deleted and curved strokes such as left-falling, right-falling, bend, hook, etc. are straightened, being removed the minutiae scientifically and reasonably, so that the fonts presented in front of people will not be too compact and make the viewer feel oppressed; instead, it is simultaneously loose and tight, with a sense of breathing, achieving the visual effect less is more. For example, if the strokes of the word "敬" are not deleted, the left half will appear overcrowded, causing imbalance between the left and right of the Chinese characters and loss of coordination. After deliberately omitting and deleting some of the strokes, it presents a simple, bright and modern font effect. In the poster of "Designer's Lecture", Jiang Hua combined the characteristics of Song typeface and boldface characters and used the principle of thin rightward strokes and thick downward strokes in Song typeface to omit the rightward strokes in Chinese characters, but still kept the triangle at the end of the strokes to help viewers recognize. Then, due to the visual characteristics of the human eye, they were supplemented into complete font forms. The overall font was concise and easy to recognize, which harmonized the visual rhythm of the picture ("Figure 8"). The series of posters designed by Bartle Bogle Hegarty for the Paralympic Games reduced the parts of Chinese characters according to the particularity of the Paralympic Games. The audience can understand the meaning behind the whole sentence according to the incomplete part, reflecting the complementary and syncretic relationship of form and meaning, and at the same time increasing the viewer's initiative, making them interested in the form of Chinese characters ("Figure 9") [5] $]^{31}$. 


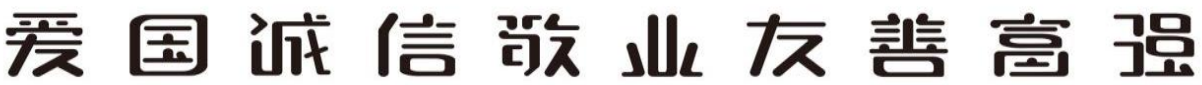

Figure 7 Self-drawn by the author.

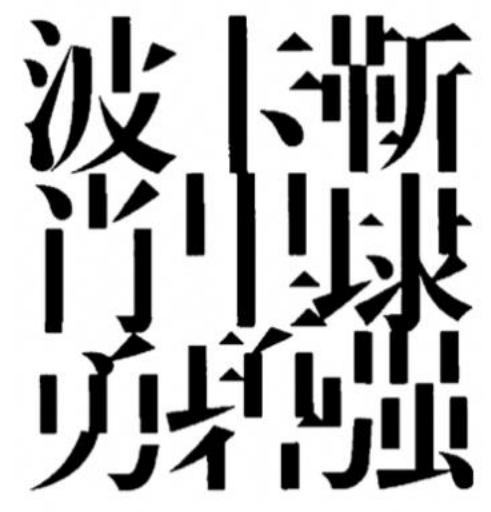

Figure 8 Jiang Hua: Designer's Lecture.

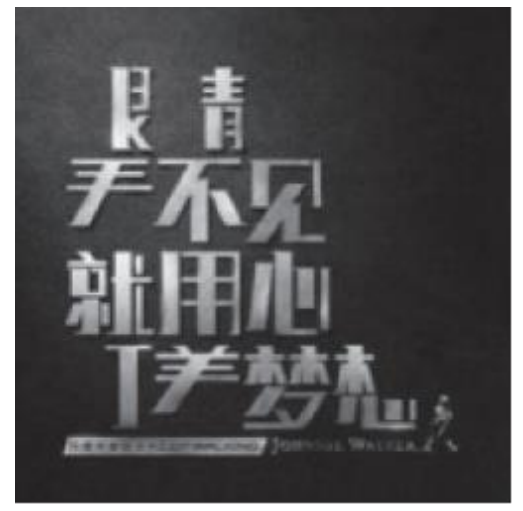

Figure 9 Bartle Bogle Hegarty: Paralympic Games series posters.

\subsection{The Continuity Constitutes the Whole - The Connection and Superposition of Strokes}

In the design of Chinese characters, the connection, joining, and intersection of strokes cannot be avoided, but when the viewer sees multiple strokes connected or intersected, they will

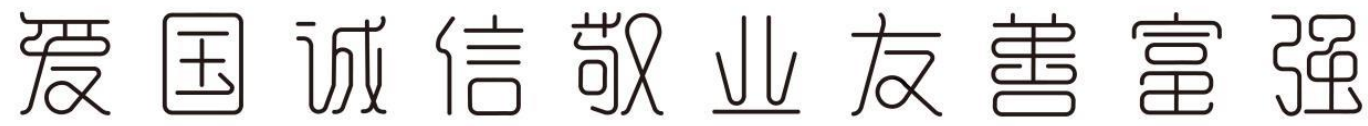

Figure $10 \quad$ Self-drawn by the author.

regard them as a whole rather than a simple stacking of several strokes. The joining of strokes can produce a tidy high-class sense, creating a concise visual atmosphere. Forcibly joining during design is forbidden; otherwise it will destroy the structure of the font itself and affect the recognition of individual fonts. While maintaining the original visual laws, it is needed to pay attention to certain characteristics or connections between fonts, and connect them through the similarity of strokes or a common stroke, so as to form a new visual relationship between strokes.

Font connection is divided into intra-character connection and inter-character connection. Intracharacter connection refers to the series connection between different strokes within a character. The Chinese character design in "Figure 10" uses the natural extension between adjacent strokes and the order of writing to connect, add extra strokes to make the font fuller, adjust the direction and length of the strokes to enhance the fluency of the font, give the characters personality and concise characteristics, and form a unique recognition effect. The inter-character connection is the morphological or structural connection of the strokes and structures between different characters. For example, "天涯海角" are sequentially connected. The four characters are neatly arranged side by side, extending and naturally merging the strokes between two adjacent characters to make them integrated. And the words "悦斓会" is a kind of dislocation connection. Two horizontal strokes of the same horizontal line are joined and the vertical strokes are borrowed to intersect them vertically and complete the joining by means of the position of the vertical line ("Figure 11"). 

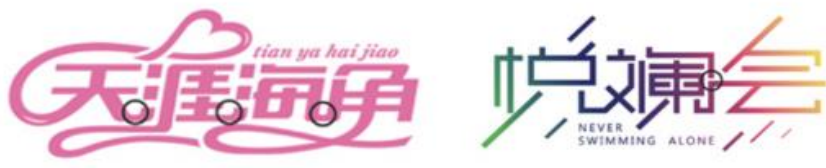

Figure 11 Tian ya hai jiao - Yue lan hui.

Arnheim mentioned in "Aesthetics Design Art Education" that in a complete picture, the point at the center is the most stable. If it is offset, it will cause the movement of the object [6] ${ }^{6}$. In the design of Chinese characters, each stroke and part has its corresponding relationship, and when they are arranged or superimposed according to a certain rule and rhythm, the viewer's eyes will gradually follow the linear flow of the adjacent dots or strokes; at this time, the viewer can experience the complex dynamics and produce the feeling of visual flow. For example, "Figure 12", it uses arrow-like graphics as stroke parts for font outlines, and overlaps them in an orderly and repeating manner to give it dynamic characteristics. At this time, the viewer's eyes will follow the direction of the arrow and move along the invisible horizontal line to form the visual perception that the Chinese characters seem to be moving. In the poster design with Beethoven's English letters as the main body, it simplified the letters into simple geometric forms and used the brightness of color and the gradual change light to form the outline trend of the characters, make the viewer's eyes move along the direction of the brightness level, and then create a sense of flow like the circle round of a record ("Figure 13").

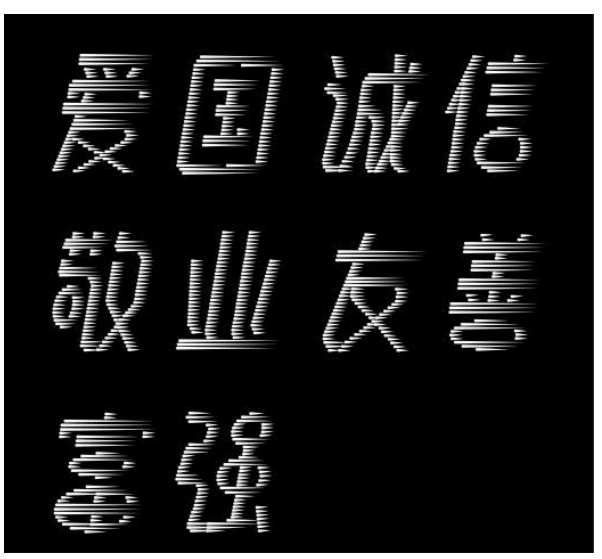

Figure 12 Self-drawn by the author. 
same skeleton structure distinctive and present simpler and more flexible characteristics.

For example, in the Chinese character design in "Figure 14", people can think of it as the character " 福", and at the same time it can be regarded as the five phrases of "爱国 (patriotism)", "诚信 (integrity)", "敬业 (dedication)", "友善 (friendliness)" and "富强 (prosperity)". The design of the Chinese character composed of two or more single characters not only retains the meaning of a single element, but also reorganizes to form a new connotation due to the close positional relationship, giving it two ways of interpretation, forming a Chinese character composed of two or more single characters with the combination of form and meaning, and conveying the meaning that patriotism is a blessing, integrity is a blessing, dedication is a blessing, friendliness is a blessing, and prosperity is a blessing. Zhang Dali's "Graphic Design in China 96" relaxes and simplifies the internal composition of characters, strengthens the upright and foursquare characteristics of the outline of characters, and forms basic geometrical morphology with simple and concise structural forms. However, the recognition of the characters is still clear, forming a strong visual impact ("Figure 15"). In the Chinese character poster "Republic of China", Han Xu merged the two characters into a common Chinese character structure, achieving the integration of visual and artistic effects and enhancing the plasticity of Chinese character design ("Figure 16").
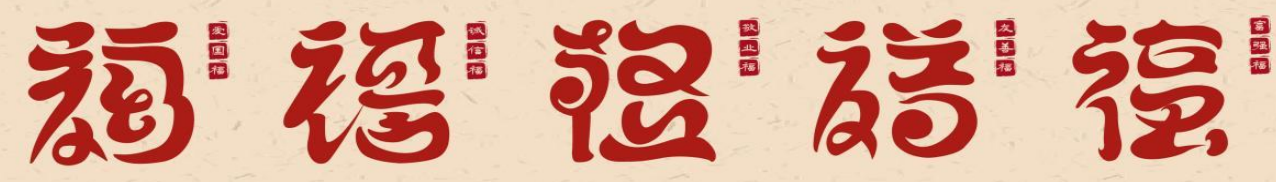

Figure 14 Self-drawn by the author.

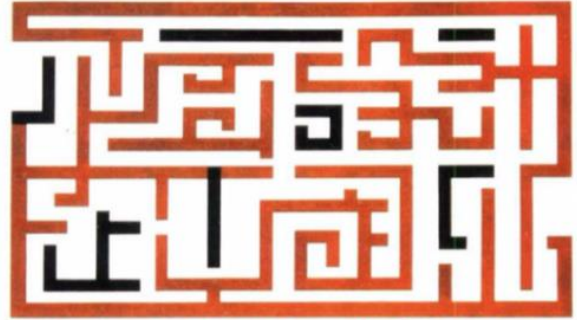

QRAABIG BEBIGN IN CHINA B

Figure 15

China 96.

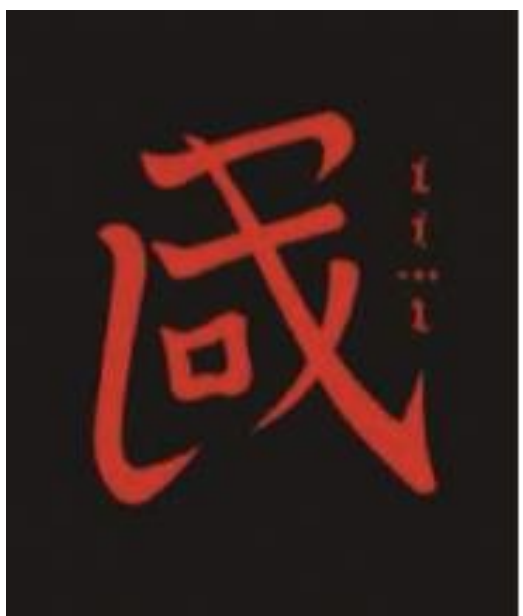

Figure 16

Han Xu's Chinese character poster.

\subsection{The Similarity Affects Grouping - The Image Conversion of Chinese Character Structures and Parts}

The design of Chinese characters can use the common factors between form, internal connection and logic to break up the original structure, and integrate new and similar elements into it to replace some parts, so as to form brand-new visual elements, conveying the characteristics of another physical image, which is equivalent to the "isostructuralism" in the design. When the viewer perceives similar elements, they will unconsciously classify them into the same kind, and even if these elements are not close in space, they will be naturally related $[4]^{97}$. It gives Chinese characters more connotations and appeal, attracts the audience, and strengthens the artistic information conveyed. However, the image replacement of certain parts of Chinese characters is not a simple element replacement, but a more suitable and perfect evolution of the structure of the characteristics of Chinese characters. During the design, it is needed to balance and coordinate the proportional relationship between the two, avoid 
competing with each other, and present the overall advantages of the combination of the two and the intuitive characteristics of replacement parts.

Each part of the characters in "Figure 17" is replaced with English letters, and the sharing of forms makes Chinese characters and letters become a whole, completely transforming the image of Chinese characters. In terms of the arrangement of strokes, such Chinese characters are no longer the original glyphs, but each part of the characters and the shape of the English letters have been maximized to match, retaining the identifying characteristics of each character. Therefore, the shape features are visually reasonable, and even if there is visual interference, it will not cause the viewer to misunderstand. For example, in the font poster "Figure 18", the low-recognition parts are replaced by the graphics related to the meaning of the characters, so that the viewer feels a strong breath of life and has interesting associations. The performance effect of both excellent pictures and texts can not only deepen the connotation of the combination of form and meaning of Chinese characters, but also give play to the infinite charm of Chinese character design.

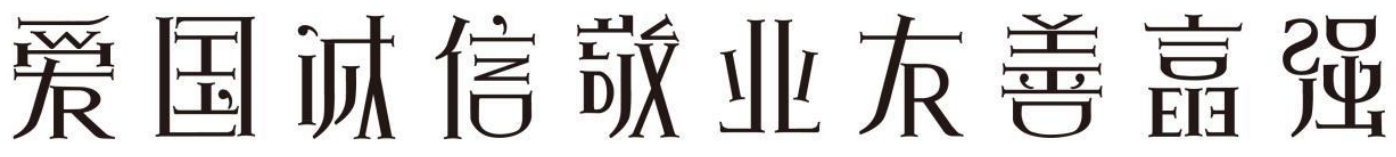

Figure 17 Self-drawn by the author.

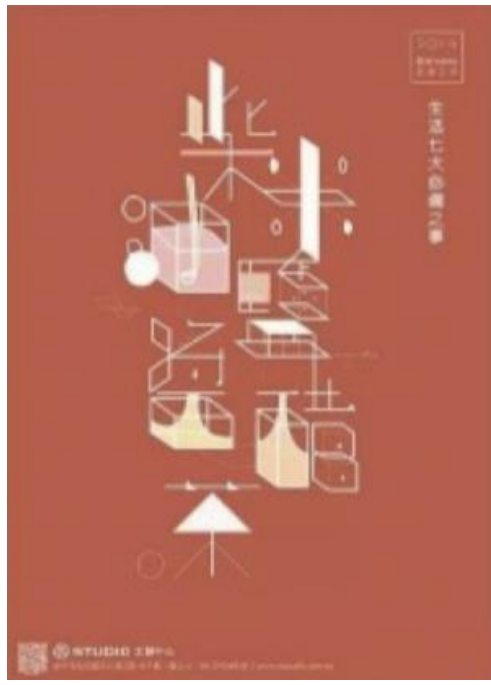

Figure 18 Seven Must-have Things in Life.

\subsection{The Figure-Ground Contrast Highlights the Main Body - The Symbiosis and Choice of the Figure and Ground}

The figure and ground in the design of Chinese characters are two wholes of equal value, which can be reversed or replaced at will. Under normal circumstances, the viewer cannot accept all the stimuli brought by the perceptual object, but will choose according to perceptual preference. The first part of a subject to be perceived is considered to be the figure and the other background part is considered to be the ground $[4]^{95}$. Therefore, the shape of the strokes of Chinese characters and the structure between the strokes can produce a wonderful conversion of positive and negative shapes, which increases the interest of the viewer when recognizing Chinese characters. Generally, the degree of distinction between the subject and the background determines the difficulty of the subject's recognition. The greater the degree of distinction, the higher the subject's recognition $[4]^{95}$. In the design of Chinese characters, if the main part is to be designed as a visual focus, it needs to have prominent features and a clear outline.

For example, in "Figure 19", it uses a thicker rectangle as the font strokes to make it as full as possible to fill the entire picture, leaving a small gap between each other, but this gap also reflects the original font. The use of thickness contrast and stroke sharing to achieve the conversion of the design form of the figure and ground makes the strokes no longer in a state of isolation and division, but a complementary and mutually dependent relationship [7] ${ }^{50}$. Another example is the font poster of Li Fojun — "Celebrating the 15th Anniversary of the Return of Macao". He designs the strokes to be thicker, rounded rectangles to fill the entire picture, and it also reflects two words "+ 五" in the gap between the two characters "澳门", thus making the audience feel curiosity, want to explore, and then think about its inner meaning ("Figure 20"). In the relationship between figure and ground, color, texture, positional relationship, etc. are also important factors to distinguish the two. In the character "旅" in the work of Makefont, 
the difference between the figure and the ground is formed according to the different colors of some of the characters of "人". Some viewers will regard the "旅" formed by the combination of "人" as the figure and the remaining black "人" as the ground, associating with the sense of tableau of crowded conditions in the tourism scene. And the comparison between figure and ground provides the best artistic interpretation of the tourism scene, which is very interesting ("Figure 21").

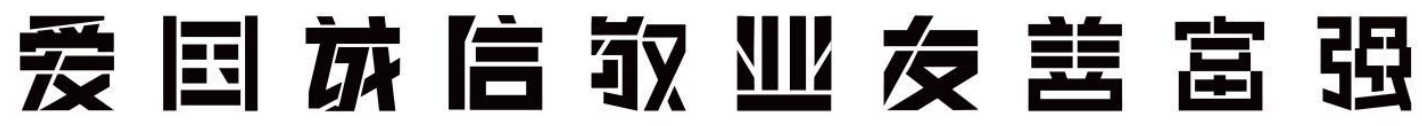

Figure 19 Self-drawn by the author.

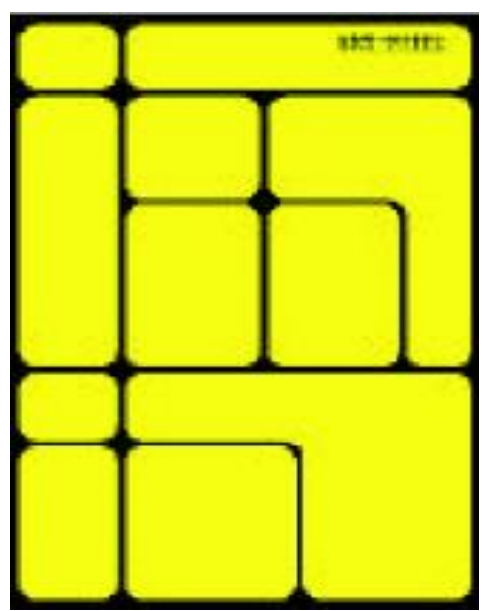

Figure $20 \quad$ Li Fojun: "Celebrating the 15th Anniversary of the Return of Macao" font poster.

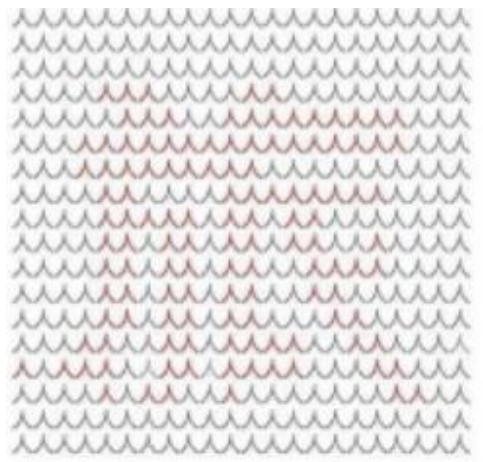

Figure 21 Font design of the Makefont.

\section{LIMITATIONS OF THE GESTALT ORGANIZATION LAW IN THE DESIGN OF CHINESE CHARACTERS}

The great disparity in the complexity of the strokes of Chinese characters and the changeful parts have led to the complex and rich changes of Chinese characters. Some Chinese characters have similar font features and weak differences, which may cause confusion and cognitive errors in recognition. However, the perception of form in the Gestalt organization law is the most essential response of human beings, and it is a high-level generalization of inner emotions. The more works that resonate with the inherent impression of the audience, the more successful they will be. Therefore, when designing, it is firstly necessary to consider the viewer's visual perception laws and psychological characteristics and analyze the factors that affect the recognition of Chinese characters. Besides, certain specifications and specific considerations should be made on the size of the font, the thickness and complexity of the stroke, the relative proportion of parts, the characteristics of the outline and the amount of actual use, etc., in order to design the external form while exuding the inner temperament of the Chinese characters, so that it integrates and unifies the form and meaning of the Chinese characters, thus making it not only meet the visual perception preference of the audience, but also help the information to be accurately conveyed.

\section{CONCLUSION}

The combination of the fault-tolerant features of Chinese characters and the Gestalt organization law makes the viewer have no difficulty in recognizing the alienated Chinese characters and relieves the visual pressure and aesthetic fatigue of the sameness when appreciating. Today, when design art is pursuing individualization, designers need to be familiar with the Gestalt organization law, carry out the creative design of Chinese characters on the basis of grasping the rules of visual perception thinking, and enrich their forms of expression, so as to make the expression of the creative design of Chinese characters more interesting and diverse and arouse the emotion and resonance of the viewers. 


\section{AUTHORS' CONTRIBUTIONS}

Kexuan Wang is responsible for Chinese characters design and wrote the manuscript, Zhixiong Huang contributed to revising and editing.

\section{REFERENCES}

[1] Wang Xin. The Application of Gestalt Law in Logo Design [J]. Design, 2017(22): 132-133. (in Chinese)

[2] Kenya Hara. Design of Design [M]. Zhu E, trans. Jinan: Shandong People's Publishing House. 2006. (in Chinese)

[3] Meng Zhaoquan. On the Originality of the Increase or Decrease of Chinese Character Strokes and Its Whole Deformation [J]. Journal of Zhongzhou University, 2015, 32(06): 95-99.(in Chinese)

[4] Liu Sha. Psychology of Design Art [M]. Beijing: Tsinghua University Press, 2006. (in Chinese)

[5] Liang Qian, He Fang. The Morphological Subtraction of Chinese Character Design Expresses the Information Perception [J]. Art and Design (Theory), 2018, 2(05): 30-32. (in Chinese)

[6] Arnheim. Art and Visual Perception [M]. Teng Shouyao, trans. Chengdu: Sichuan People's Publishing House, 2019. (in Chinese)

[7] $\mathrm{Hu}$ Yanzhen, Liu Yiwei. Analysis of the Fuzzy Design Language in Chinese Font Design $[\mathrm{J}]$. Beauty \& Times (Part I), 2017(05): 49-51. (in Chinese) 